\title{
State of the art in the teaching of digestive ultrasound
}

\author{
Giovanni Maconi ${ }^{1}$, Mafalda Sousa ${ }^{2}$
}

${ }^{1}$ Gastroenterology Unit, ASST-Luigi Sacco University Hospital, Milan, Italy, ${ }^{2}$ Gastroenterology Unit, Centro Hospitalar de Vila Nova de Gaia e Espinho, Vila Nova de Gaia, Portugal

\begin{abstract}
Gastrointestinal ultrasound (GIUS) has been increasingly used for the management of gastrointestinal disorders due to its potentialities as a cheap, widely available and safe examination with comparable accuracy to other techniques such as Computed Tomography or Magnetic Resonance Imaging. However, some limitations have been stated to justify its underuse such as its dependency on the operator, small field of view and penetration and need of expertise. However, most of these limitations are inherent to any technique. The main problems with GIUS are lack of standardized definitions, adequate training programs and measures to assess the quality. The definition of universally applicable standardized measurements, the implementation of an internationally recognized curriculum as well as an agreed set of core competencies and the assessment of performance will hopefully overcome its potential limitations and implement its utilization universally in clinical practice.
\end{abstract}

Keywords: gastrointestinal ultrasound; training; quality; standardization

\section{Introduction}

Digestive ultrasound (US) or gastrointestinal ultrasound (GIUS) is not a new technique. The first description of its usefulness to assess the bowel wall layers and pathologic changes dates more than 40 years ago [1]. Since then, technological US advancements and increased skills of sonographers, most of them gastroenterologists, broadened its use to diagnosis and monitoring several intestinal diseases, in particular inflammatory bowel disease (IBD). Systematic reviews and meta-analyses have shown that GIUS, Computed Tomography scan (CT) and Magnetic Resonance Imaging (MRI) have

Received 02.10.2019 Accepted 14.12.2019

Med Ultrason

2020, Vol. 22, No 1, 71-74

Corresponding author: Giovanni Maconi, MD

Gastroenterology Unit. ASST-Luigi Sacco

University Hospital - Milan, Italy

Hospital, Via GB Grassi, 74,

20157 Milan, Italy

Phone: $++39 / 2 / 39043164$

Fax: $++39 / 2 / 3942232$

E-mail: giovanni.maconi@unimi.it similar accuracy for the evaluation of IBD [2-5], and currently GIUS is recommended by several international guidelines as the first examination in patients with abdominal complaints to detect appendicitis, diverticulitis, bowel occlusion and inflammatory bowel diseases [6-9]. The advantages of GIUS over other imaging techniques are its relatively low cost, the widely available equipment and potential prompt use and the lack of contraindications or harmful conditions such as ionizing radiation or claustrophobia [10]. Yet, despite its high accuracy and clear advantages, the incorporation of GIUS into routine gastroenterology practice remains somehow inconsistent and still underused and even considered with skepticism [11]. The main reasons of this could be attributed to some limitations of GIUS compared to other diagnostic modalities, such as the small field of view and penetration (in particular in oversize and obese patients), limited standardization and reproducibility of scan protocols, lower capacity for retrieval and re-evaluation of archived images, and, not least, the superior "time to remuneration" ratio of this technique compared to the MRI or CT scan [10]. However, probably, one of the most stated limitations is that it is considered "operator dependent" 
and of value only in super-expert hands. This limitation is poorly founded, as the performance and interpretation of every diagnostic test are, without exception, "operator dependent".

The real limitations of GIUS are probably mainly related to the lack of international consensus regarding the standardization of the technique, the lack of training and performance standards and the lack of quality indicators (Table I).

The present review will discuss the present limitations of GIUS, in particular the need of a standardized intestinal ultrasound terminology, of a proper training curriculum and quality assessment, and will try to provide some hints on the future directions of this technique.

\section{The standardization of intestinal ultrasound}

To explain the need of standardization, for instance, though we know that a bowel stricture is sonographically defined as a narrowing of the lumen, coupled with thickening of the walls and prestenotic dilatation, the literature counts 10 different definitions of strictures in Crohn's disease [12]. Thus, it is not surprising if in a recent study, which assessed the interobserver agreement among 6 blind expert operators during scanning $15 \mathrm{CD}$ patients, with no preliminary formal didactic session, poor results were obtained in the detection of stenosis and of prestenotic dilatation [9].

To overcome this important limitation, an international team of experts in GIUS, with the endorsement of the European Federation of the Societies of Ultrasound in Medicine and Biology (EFSUMB), was created in 2016, with the aim to standardize definitions in IBD and other gastrointestinal diseases and to promote its use in a clinical setting [7]. The implementation of these universally applicable standardized measurements will provide a basis for measuring, evaluating and improving performance and quality of care in GIUS [10].

\section{Training in gastrointestinal ultrasound}

An internationally recognized curriculum, as well as an agreed set of core competencies in GIUS are still lacking. The training in US should be part of the medical student curriculum, or at least, of the various specializations. Actually, even radiological specialization, in some instances, lacks appropriate training for GIUS. Therefore, usually trainees themselves take on the burden of pursuing a comprehensive education [10].

Overall, the learning process of GIUS - as for every other issues - may include several steps as defined by Edward Thorndike, an American psychologist and known
Table I. Present limitations of GIUS and future directions

\begin{tabular}{ll}
\hline $\begin{array}{l}\text { Current limitations } \\
\text { in GIUS }\end{array}$ & Future directions \\
\hline $\begin{array}{l}\text { Lack of standard } \\
\text { definitions }\end{array}$ & $\begin{array}{l}\text { Implementation of universally appli- } \\
\text { cable standardized measurements }\end{array}$ \\
Lack of training & $\begin{array}{l}\text { Internationally recognized curriculum } \\
\text { and agreed set of core competencies }\end{array}$ \\
$\begin{array}{l}\text { Lack of quality } \\
\text { assessment }\end{array}$ & $\begin{array}{l}\text { Objective criteria for evaluating GIUS } \\
\text { competence }\end{array}$ \\
\hline
\end{tabular}

GIUS - Gastrointestinal ultrasound

as the father of educational psychology, who lived more than 100 years ago [13]. His learning process specified certain ingredients:

1) Motivation: this implies a strong determination, concentration, clear objectives and well-founded reasons to learn;

2) Exercise: things that are repeated are better remembered or better performed;

3) Emotional reactions: the learning is reinforced when it is accompanied by pleasant or satisfying sensations (e.g., detecting a disease); weakens when it is associated with unpleasant or frustrating sensations;

4) Plan priorities: things learned first often create a strong and almost indelible impression, considering that this also means that bad habits learned early are hard to die;

5) Intensity: a vivid, exciting or engaging experience is more effective to learn than a boring exercise routine (e.g. a student learns more from the "real life" than from simulation).

From a practical point of view, the learning process of US involves image interpretation, that is mainly cognitive and image acquisition, that is mainly behavioral. While the first is merely a theoretical step, the second is a practical context that is achieved by means of a hands-on session and daily performance activity. Both these steps are mandatory to reach appropriate skills and competence.

Competence in US has changed in the last decade, and is still changing now. Point of Care Ultrasound (POCUS) is an example. POCUS is an expanding field in medicine, that focuses on the training of operators to answer specific, clinically pertinent questions. Pattern recognition and expertise within a limited remit of US skills applied to specific clinical contexts, can provide acceptable diagnostic accuracy without the necessity for "full diagnostic level of knowledge and expertise" in US. This paradigm represents a shift from fear of false reassurance and missed findings of significance, to a recognition that the appropriate use of US improves diagnostic accuracy over clinical examination alone. 
Given the increasing interest in particular among non-experts in using US examination in daily practice, there is a concern to ensure high-quality US services in every setting. Consequently, several international training boards have issued recommendations regarding training and certification of non-radiologists executing USbased procedures [14]. For example, a statement from the American Institute of Ultrasound in Medicine specified that trainees in abdominal US should have evidence of being involved in the performance, evaluation, interpretation and reporting of at least 500 diagnostic US examinations and recommends a minimum of 300 diagnostic abdominal US per year to maintain the appropriate skills. An EFSUMB document states that gastroenterologists should execute 5-10 examinations per week, reaching a minimum of 300 examinations before performing US unsupervised and in addition, a yearly minimum of 100 procedures is suggested $[15,16]$.

So, the number of examinations seems a crucial issue in every training program but we have to consider the learning curve and its slope. Little data is available regarding digestive US and we do not know exactly the slope for specific issues, namely the minimum for each specific clinical question for POCUS. Kim et al, dealing with the learning curve for acute appendicitis, included 3 residents with no experience in US examinations that completed a dedicated 1-day course consisting of a 1-hour lecture, 3 hours of hands-on training and 2 hours self-study. The diagnostic agreement between the emergency US findings and the final diagnoses, was assessed using Cohen's kappa coefficient. Over a population of 266 patients of whom 127 patients $(47.7 \%)$ had acute appendicitis, the three residents showed a low concordance (kappa value of $<0.6$ ) in the first 20 cases but after, the overall kappa value increased to 0.84 (95\% CI: 0.77 0.92) [17]

In an preliminary study the performance of 4 sonographers ( 2 with experience in US but not GIUS and 2 without experience in US or GIUS) was assessed and compared to an experienced sonographer in GIUS [18]. It was shown that sonographers with experience in US had a shorter learning curve in detecting pathological bowel wall thickening, enlarged lymph-nodes and abnormal dilatation of the bowel. After 50 examinations, physicians trained in abdominal US had a variable and increasing agreement with the experienced sonographer, from the first to the third clusters of patients, with $\mathrm{k}$ values of 0.82-0.94, 1 and 1 for detection of increased wall thickness; $0.90-1,1$ and 1 for abnormal dilatation; 0.69-0.81, 0.72-1 and 0.89-1 for enlarged mesenteric lymph-nodes. Physicians without experience in US had an increasing agreement with the experienced sonographer: 0.60-0.76,
0.86-0.94 and 1 for bowel wall thickness; 0.72-0.90, 1 and 1, for abnormal bowel dilatation, 0.37-0.46, 1, and 0.93-1 for enlarged mesenteric lymph-nodes. This shows that although learning curves are different between individuals, even non-experienced sonographers can achieve good results after an adequate number of examinations and proper training.

\section{Quality assessment}

But when can we consider ourselves at the top of the learning curve? When can we be considered competent? How is it possible to define the quality assessment? These are important unsolved questions. An attempt to create objective criteria for evaluating our US competence has been proposed by an international consensus [19]. Seven key elements of the ultrasound examination were identified: 1) Indication for the examination, 2) Applied knowledge of ultrasound equipment, 3) Image optimization, 4) Systematic examination, 5) Interpretation of images, 6) Documentation of examination and 7) Medical decision making.

This Objective Structural Assessment of Ultrasound (OSAUS) was used to assess the competence in US in a recent study by Todsenet al [20]. Twenty-four physicians were included, representing novices, intermediates and experts in POCUS. Four different surgical patient cases were scanned and all US examinations were video-recorded and assessed by 2 blinded radiologists using OSAUS. Construct validity was examined by comparing performance scores between the groups and by correlating physicians' OSAUS scores with diagnostic accuracy. The study showed that OSAUS was able to significantly discriminate between the performances of the sonographers.

How to improve and maintain competence is another important issue. It is generally advisable to attend dedicated and accredited meetings, especially with hands-on training, and perform training periods in dedicated centres with a high volume of examinations and with complex cases containing varied pathologies. National and international sonographic societies should support these opportunities. And last, but not least, websites where webinars, case challenges, literature news, resource libraries and atlas of images are available, greatly improve knowledge in ultrasound.

\section{Conclusion and future directions}

The use of GIUS inevitably increases our skills and affects our workload in general over time. There is a pressing need to use standardized ultrasound terminol- 
ogy, to provide training and to continuously assess the quality of our sonographic performances. This will allow multicenter studies that could give us answers to important areas such as follow up and monitoring in Crohn's disease and Ulcerative Colitis.

\section{Conflict of interest: none}

\section{References}

1. Holt S, Samuel E. Grey scale ultrasound in Crohn's disease. Gut 1979;20:590-595.

2. Panés J, Bouzas R, Chaparro M, et al. Systematic review: the use of ultrasonography, computed tomography and magnetic resonance imaging for the diagnosis, assessment of activity and abdominal complications of Crohn's disease. Aliment Pharmacol Ther 2011;34:125-145.

3. Dong J, Wang H, Zhao J, et al. Ultrasound as a diagnostic tool in detecting active Crohn's disease: a meta-analysis of prospective studies. Eur Radiol 2014;24:26-33.

4. Fraquelli M, Colli A, Casazza G, et al. Role of US in detection of Crohn disease: meta-analysis. Radiology 2005;236:95-101.

5. Horsthuis K, Bipat S, Bennink RJ, Stoker J. Inflammatory bowel disease diagnosed with US, MR, scintigraphy, and CT: meta-analysis of prospective studies. Radiology 2008;247:64-79.

6. Dirks K, Calabrese E, Dietrich CF, et al. EFSUMB Position Paper: Recommendations for Gastrointestinal Ultrasound (GIUS) in Acute Appendicitis and Diverticulitis. Ultraschall Med 2019;40:163-175.

7. Maconi G, Nylund K, Ripolles T, et al. EFSUMB Recommendations and Clinical Guidelines for Intestinal Ultrasound (GIUS) in Inflammatory Bowel Diseases. Ultraschall 2018;39:304-317.

8. Panes J, Bouhnik Y, Reinisch W, et al. Imaging techniques for assessment of inflammatory bowel disease: joint ECCO and ESGAR evidence-based consensus guidelines. J Crohns Colitis 2013;7:556-585.

9. Calabrese E, Maaser C, Zorzi F, et al. Bowel Ultrasonography in the Management of Crohn's Disease. A Review with
Recommendations of an International Panel of Experts. Inflamm Bowel Dis 2016;22:1168-1183.

10. Atkinson NS, Bryant RV, Dong Y, et al. WFUMB Position Paper. Learning Gastrointestinal Ultrasound: Theory and Practice. Ultrasound Med Biol 2016;42:2732-2742.

11. Asthana AK. Intestinal ultrasound in inflammatory bowel disease. J Gastroenterol Hepatol 2016;31 Suppl 1:21-22.

12. Coelho R, Ribeiro H, Maconi G. Bowel Thickening in Crohn's Disease: Fibrosis or Inflammation? Diagnostic Ultrasound Imaging Tools. Inflamm Bowel Dis 2017;23:2334.

13. Thorndike EL. Human learning. New York: The Century Co; 1931.

14. Pinto J, Azevedo R, Pereira E, Caldeira A. Ultrasonography in Gastroenterology: The Need for Training. GE Port J Gastroenterol 2018;25:308-316.

15. AIUM. Training guidelines. Training Guidelines for Physicians Who Evaluate and Interpret Diagnostic Abdominal/ General Ultrasound Examinations. Official Statement n.d. Accessed September 13, 2019. Available at: https://www. aium.org/resources/viewStatement.aspx?id=47

16. Education and Practical Standards Committee, European Federation of Societies for Ultrasound in Medicine and Biology. Minimum training recommendations for the practice of medical ultrasound. Ultraschall Med 2006;27:79105.

17. Kim J, Kim K, Kim J, et al. The learning curve in diagnosing acute appendicitis with emergency sonography among novice emergency medicine residents. J Clin Ultrasound 2018;46:305-310.

18. Monteleone M, Friedman AB, Furfaro F, Dell'Era A, Bezzio C, Maconi G. P139 The learning curve of intestinal ultrasonography in assessing inflammatory bowel disease preliminary results. J Crohn Colitis 2013;7:S64.

19. Tolsgaard MG, Todsen T, Sorensen JL, et al. International multispecialty consensus on how to evaluate ultrasound competence: a Delphi consensus survey. PloS One 2013;8:e57687.

20. Todsen T, Tolsgaard MG, Olsen BH, et al. Reliable and valid assessment of point-of-care ultrasonography. Ann Surg 2015;261:309-315. 